\title{
Les effets des exportations d'armements chinois sur la violence régionale
}

\section{R. Bates Gill}

\section{OpenEdition}

\section{Journals}

Édition électronique

URL : http://journals.openedition.org/conflits/121

DOI : $10.4000 /$ conflits. 121

ISSN : $1777-5345$

Éditeur :

CCLS - Centre d'études sur les conflits lilberté et sécurité, L'Harmattan

Édition imprimée

Date de publication : 17 janvier 1991

ISSN : 1157-996X

\section{Référence électronique}

R. Bates Gill, «Les effets des exportations d'armements chinois sur la violence régionale », Cultures \& Conflits [En ligne], 04 | hiver 1991, mis en ligne le 31 décembre 2002, consulté le 30 mars 2021. URL: http://journals.openedition.org/conflits/121 ; DOI : https://doi.org/10.4000/conflits.121

Ce document a été généré automatiquement le 30 mars 2021.

Creative Commons License 


\section{Les effets des exportations d'armements chinois sur la violence régionale}

\section{R. Bates Gill}

1 Il y a beaucoup de pays qui vendent des armements à d'autres pays. Mais quand la Chine les vend, la presse tend à chercher querelle à la Chine, et la Chine est en vedette Comment se fait-il que certains s'en prennent sans cesse à la Chine à ce sujet? Wu Xueqian, ancien ministre chinois des Affaires étrangères.

2 Ce commentaire, parmi bien d'autres ${ }^{1}$, traduit l'attitude assez défensive de l'oligarchie chinoise sur sa politique d'exportations d'armes. Mais, en dépit de ces protestations, la République Populaire de Chine $^{2}$ a atteint, pendant les années quatre-vingts, une certaine notoriété en tant que marchande d'armes : un observateur distingué, n'a-t-il pas déclaré que la Chine était un "éléphant solitaire dans le commerce des armes, qui fournit des armes quasiment sans tenir compte des conséquences en matière de politique ou de sécurité" ${ }^{3}$. Même si une telle déclaration est un peu exagérée, il reste vrai que la RPC a mené, ces dernières années, une campagne agressive pour élargir sa part du commerce international des armes, ce qui explique que la question des exportations d'armes chinoises revienne souvent dans l'actualité ; d'ailleurs la liste des clients de la Chine continue de s'allonger. Citons parmi d'autres pays: l'Iran, l'Irak, l'Arabie Saoudite, la Syrie, la Thaïlande, la Birmanie, l'Algérie.

3 Cette publicité récente autour des exportations d'armes chinoises ne doit pas faire oublier que la RPC pratique le commerce des armes depuis sa création en 1949. Si les armes exportées par la RPC n'ont jamais égalé - en matière de qualité ou de quantité celles d'autres grandes puissances: l'Union soviétique, les états-Unis, la France, le Royaume-Uni, il faut savoir que, depuis 1951, la RPC a toujours figuré parmi les cinq plus grands fournisseurs d'armements au tiers-monde (voir tableau 1). Les exportations militaires chinoises ont d'ailleurs modifié d'une manière significative deux grandes "batailles" de la Guerre froide; la guerre en Corée dans les années cinquante et la Guerre du Vietnam dans les années soixante/soixante-dix. A la fin des années soixante- 
dix, et au début des années quatre-vingts, la RPC a beaucoup augmenté ses exportations militaires, entre 1980-1990 la Chine a même dépassé le Royaume-Uni pour devenir le quatrième fournisseur d'armements du tiers-monde. Les cinq plus grands fournisseurs d'armements vers le tiers-monde 1951-19904 1951-1959 1960-1969 1970-1979 1980-1990

\section{USA URSS URSS URSS}

\section{UK USA USA USA}

\section{URSS UK France France}

\section{France France UK Chine}

5.Chine Chine Chine UK

Ainsi la Chine a augmenté les volumes et la qualité des armes qu'elle exporte, et a élargi le nombre de ses clients. Elle a franchi en outre un seuil très controversé en fournissant de la technologie nucléaire à des pays tels que l'Iran, le Pakistan, et l'Algérie. Tout cela mérite largement de retenir l'attention des observateurs de la conflictualité régionale et mondiale. A partir de trois régions troublées auxquelles la Chine fournit des armes: l'Asie du Sud-Ouest, l'Asie du Sud, et l'Asie du Sud-Est, cet article tentera de répondre à deux questions: Comment les conflits régionaux influencent-ils les exportations d'armes chinoises ? Comment sont-ils influencés par les ventes d'armements chinois? Il essayera également de faire la lumière sur la nature de la politique d'exportations d'armes de la Chine. Mieux comprendre ces questions exige tout d'abord de restituer l'évolution historique de la Chine en tant que marchande d'armes dans le contexte du développement global du commerce des armes. Les exportations d'armements chinois : développements contemporains La Chine est un bon modèle pour comprendre les changements qui ont marqué le commerce des armes depuis les années soixante-dix. Tout d'abord en augmentant la quantité de ses armes exportées, en améliorant leur qualité, et en diversifiant sa clientèle, la Chine a contribué à la prolifération des armes dans le monde. Ainsi la part chinoise de la valeur totale des armements exportés vers le tiers-monde a nettement augmenté puisqu'elle passe de 1,8\% pendant la période 1970-1979, à 7,5\% pendant la période $1986-1990^{5}$. De plus, la Chine est disposée à transférer des armes relativement avancées, conçues pour l'exportation. Enfin, le poids de la Chine dans ce type de commerce symbolise aujourd'hui la multiplication de sources d'armements dans le monde; la Chine exporte aussi des armements vers les pays avec lesquels elle n'avait pas des relations très étroites, voire même à des clients de l'URSS. Reprenons ces divers éléments.

La croissance rapide des quantités d'armements exportées par la Chine particulièrement notable est le premier fait qu'il faut souligner. Le tableau 2 compare la valeur des exportations d'armements des Etats-Unis, de l'Union soviétique, et de la Chine vers le tiers-monde de 1976 à 1990. Il est tout à fait remarquable que la valeur des armements chinois exportés au cours des cinq dernières années (1986-1990: 7,569 milliards $/ \$^{6}$ ) représente presque cinq fois la valeur de la première période de cinq ans (1976-1980 : 1,579 milliard/\$), et $160 \%$ la valeur de la deuxième période de cinq ans (1981-1985 : 4,655 milliards/\$). En effet, la valeur totale enregistrée pour la période 1986-1990 dépasse la valeur des quatorze années précédentes (1972-1985: 7,381 milliards/\$). Tableau 2.

11 Comparaison de la valeur des armements exportés vers le Tiers-Monde par la Chine, l'Union Soviétique et les Etats-Unis, 1976-19907. L'augmentation qualitative des armements chinois - deuxième fait à souligner - est aussi remarquable. En matière de 
qualité, nous notions plus haut que les armements chinois ne sont pas aussi perfectionnés que ceux d'autres grandes puissances. Mais, la Chine est disposée à offrir à ses clients ce qu'elle produit de mieux. Depuis l'époque où elle dépendait de l'Union soviétique, l'industrie militaire de la Chine a beaucoup progressé, notamment par le biais du "retrofitting"8, ce qui permet d'offrir des armements encore plus intéressants aux clients dans le tiers-monde. Les progrès des ingénieurs militaires chinois sont également remarquables dans le domaine des missiles et des avions, mais aussi dans celui de la technologie nucléaire ${ }^{9}$.

Troisième remarque, au lieu de s'en tenir à ses clients traditionnels, la Chine, comme d'autres fournisseurs d'armements, a cherché une nouvelle clientèle - trouvant même des acheteurs parmi les pays qui étaient encore, il y a peu de temps, des adversaires politiques. Par exemple, l'exportation des armements chinois aux ex-clients soviétiques - la Syrie et l'Irak - est frappante. Fait plus remarquable encore, la Chine a exporté 60 missiles CSS-2 en Arabie Saoudite, à un moment où les deux pays n'avaient pas encore établi de relations diplomatiques, ce qui signifiait que pour la Chine, l'Arabie Saoudite n'était à ce moment-là qu'un pays "féodal". C'est également grâce au commerce des armes que les relations RPC-Thaillande se sont transformées en une quasi-alliance, alors que la Chine a soutenu le mouvement communiste thailandais contre le gouvernement à Bangkok jusqu'en 1978. Deux autres pays de l'Asie du Sud-Est - la Birmanie et l'Indonésie - considérèrent la Chine comme une menace grave dans la région, depuis les années soixante, et cependant, ces deux pays ont importé des armes chinoises en 1990 pour la première fois. Depuis 1949, la RPC a fourni plusieurs types d'aide militaire entraînement, conseillers, projets de construction militaire (aéroports, arsenaux navals, usines), armes personnelles, accords de production sous licence, matériel lourd - à au moins 40 pays et 29 mouvements révolutionnaires et minorités combattantes. Mais le secret qui entoure le commerce des armes et les différences de méthodes employées par les chercheurs et les services de renseignement pour établir leurs statistiques, explique que les chiffres sont probablement sous-estimés. Tentons, sur la base de sources fables, de proposer une appréciation plus complète (mais sans doute non exhaustive) des exportations d'armements de la Chine depuis 1949. Pendant les années cinquante et soixante, la RPC a joué un rôle particulièrement actif en tant que fournisseur d'armements aux mouvements révolutionnaires du tiers-monde. Dans les années soixante-dix, Pékin a fourni une aide militaire à divers acteurs internationaux tels que Israël et l'OLP, y compris le groupe Abou Nidal, le Zimbabwe et l'Afrique du Sud, la Corée du Nord et les Etats-Unis, l'Iran et l'Irak, même à Taïwan et aux "contras" du Nicaragua. La Chine a entrâné 23 mouvements de libération, procuré des armes individuelles (pistolets à main, mitraillettes, grenades, carabines) à peut-être 45 clients, et elle s'est engagée à construire plusieurs bases militaires dans le tiers-monde. En matière d'armements lourds, des missiles chinois sont installés dans 14 pays, 26 pays ont reçu des blindés et de l'artillerie chinoise ; 20 pays ont importé des avions militaires chinois ; 23 pays ont reçu des navires de guerre chinois (voir tableau 3). L'exportation d'armements chinois : inventaire géographique $\mathrm{Si}$, jusqu'au milieu des années quatrevingts, peu de recherches ont porté sur les exportations d'armes chinoises; depuis 1985 , un certain nombre d'études universitaires ont attiré à juste titre l'attention sur ce phénomène ${ }^{10}$. Le texte ci-après éclaire cette question. A partir de trois conflits dans lesquels la Chine est un important fournisseur d'armes: le conflit cambodgien en Asie du Sud-Est, la rivalité Inde-Pakistan en Asie du Sud, et la guerre Iran-Irak en Asie du Sud-Ouest, il cherche à analyser les relations entre les armements exportés et la 
violence régionale : Quel est l'effet des armements chinois sur les conflits régionaux, et celui des conflits régionaux sur les exportations d'armements chinois? Les transferts d'armements en Asie du Sud Est Les réalignements géopolitiques pendant les années soixante-dix ont modifié la nature des armements chinois exploités. En Asie du Sud-Est, le rapprochement sino-américain et l'hostilité latente entre la RPC et l'URSS ont contribué à miner les relations RPC/Vietnam. En revanche, à la même période, la Chine a commencé à soutenir les voisins de Hanoi et à réduire son soutien aux mouvements communistes en Thaïlande, en Birmanie, et en Malaisie. Après l'invasion et l'occupation vietnamiennes du Cambodge, la Chine et la Thaïlande ont cherché à établir des liens stratégiques contre la menace vietnamienne - liens qui se traduisaient de plus en plus par des transferts d'armements vers la Thaïlande et les Khmers rouges. -Thaïlande, Khmers rouges Bien qu'on ait prétendu qu'ils soient plus anciens", les transferts d'armements chinois ont commencé en 1985. Depuis cette date, Pékin offre un grand choix d'armements lourds: artillerie munitions transports blindés, avions, chars d assaut, sous-marins, missiles. La première transaction attestée avec la Thaïlande se situe vers la fin 1985. Ce transfert - en réalité un don - comprenait des pièces d'artillerie à longue portée de $130 \mathrm{~mm}$, des pièces antiaériennes de $37 \mathrm{~mm}$, des canons antichars de $85 \mathrm{~mm}$, et 24 chars d'assaut T 59. Un an plus tard, en janvier 1987, un accord entre les deux partis tut signé selon lequel la Chine fournirait de nouveaux armements à la Thaïlande en trois étapes. Durant la première phase, la Thaïlande a reçu 30 chars d'assaut T-69 armés d'un canon de $100 \mathrm{~mm}$, et une option sur 70 chars d'assaut. Ce transfert incluait aussi plus de 400 transports blindés type 531, des batteries d'artillerie antiaérienne de $37 \mathrm{~mm}, 3000$ grenades lance-fusées, et une quantité non-spécifiée de munitions de $130 \mathrm{~mm}^{11}$. De plus, la RPC offrait, à bas prix, des missiles antiaériens, un sous-marin de la classe "Roméo", et des avions de chasse F- $7^{12}$. Cette première phase active s'est développée dans le cadre d'un rapprochement diplomatique marqué par des discussions fréquentes à très haut échelon où se dessinait un consensus stratégique à l'égard de la sécurité régionale. En mars 1988, la deuxième phase d'achats a porté sur 23 chars d'assaut T-69 avec des canons rayés de $105 \mathrm{~mm}, 360$ transports blindés, un système de radar antiaérien, un système de radar pour missiles sol-air téléguidé, et des munitions de $130 \mathrm{~mm}$. Peu après, en juillet, le gouvernement thaïlandais a annoncé son intention d'acquérir quatre frégates "Jianghu", et 18 pièces d'artillerie antiaérienne de $37 \mathrm{~mm}$. En février 1991 une de ces quatre frégates est sortie du chantier naval de Shanghai ; les trois autres frégates seront livrées dans les premiers mois de 1992. En 1992, la marine thaillandaise recevra deux frégates supplémentaires de la classe "Jiangdong"13, plus grandes et plus modernes.

La troisième phase, qui correspond aux années 1989 et 1990, fut caractérisée par un commerce d'armements encore plus important. Début 1989, les militaires thaïlandais cherchaient à acquérir une escadrille d'avions de chasse F-7, trois sous-marins, des missiles, des chars d'assaut et de transports blindés supplémentaires. Mais, à la fin de l'année, après maintes difficultés, les Thaïlandais décidèrent de ne pas choisir le F-7 ${ }^{14}$. Un an plus tard, en août 1990, le conseil de cabinet thaïlandais a approuvé l'achat de 50 missiles anti-navires de type C-801. Ces missiles seront probablement installés sur les frégates "Jianghu" et "Jiangdong" livrées à la Thaïlande. Le tableau 4 offre une vision plus détaillée des principales exportations d'armements de la Chine vers la Thaïlande ${ }^{15}$ Tableau 4 d'armements sino-thaï se sont principalement développés pour répondre à la menace 
expansionniste vietnamienne (soutenue par les Soviétiques), c est dire qu ils reposent sur des intérêts convergents des deux pays. Mais la Chine qui sponsorise toutes les armées anti-vietnamiennes s'intéressait également aux combattants Khmers rouges qui constituent la plus grande, la plus active, la mieux entraînée et la plus puissante des factions de la résistance cambodgienne, et va en devenir le principal fournisseur. Elle les a tout d'abord armés, de 1975 à 1978, pendant la période chaotique où Pol Pot régnait sur les Cambodgiens, puis après 1979, la Chine a soutenu leur campagne antivietnamienne dans le cadre de la guerre civile au Cambodge. Les armes fournies étaient pour l'essentiel adaptées à la guérilla: fusils, revolvers, mitrailleuses, munitions, bazookas, fusées, artillerie légère antichars. Dès 1984, la Chine livrait assez d'armements pour équiper 40000 combattants $^{17}$. Mais, en 1990, pour la première fois la Chine a envoyé de l'armement lourd - 24 chars d'assaut T-59 - aux Khmers rouges ${ }^{18}$. Ces chars, ainsi que d'autres armements, furent livrés aux Khmers rouges, malgré les promesses d'un arrêt des fournitures d'armes à la résistance cambodgienne, faites par Pékin en septembre 1990. On ne sait si ces derniers transferts concernaient des armements déjà "en voie de livraison" ou portaient sur de nouvelles commandes. Quoi qu'il en soit, à la suite du traité de paix cambodgien, la Chine ne livre plus autant d'armes aux Khmers rouges. En conclusion, on doit remarquer la présence croissante de la Chine en tant que fournisseur d'armements dans la région. Laos, Birmanie, Indonésie Depuis 1990, la Chine exporte des armements au Laos, en Birmanie, et à l'Indonésie. La Chine n'avait jamais exporté d'armements au Laos, mais en 1990, le gouvernement laotien a accepté deux avions de transport Y 12 chinois ${ }^{19}$. Le commerce des armes entre Pékin et la Birmanie d'une part, et Pékin et l'Indonésie d'autre part, est encore plus intéressant à considérer si l'on se souvient de leurs relations passées; ces pays se considérèrent comme des ennemis pendant la plus grande partie de la période après-guerre. Par exemple, de 1965, date de la chute de Sukarno en Indonésie, jusqu'à l'été 1990, les relations diplomatiques sino-indonésiennes ont été interrompues. Mais tout de suite après le rétablissement des relations officielles entre Pékin et Jakarta, les deux pays ont entamé des négociations sur la vente de navires de guerre chinois à $\mathrm{l}^{\prime}$ Indonésie ${ }^{20}$. Les relations entre la RPC et la Birmanie étaient mauvaises à cause du soutien actif par la Chine des communistes birmans qui luttaient contre le gouvernement de Rangoon. Et pourtant, après la visite à Pékin en 1989 d'une délégation militaire birmane de haut rang, la RPC a commencé à exporter des armements, première partie d'une plus grande transaction estimée à 1 milliard de dollars. Cette transaction incluait des systèmes de radar d'artillerie antiaérienne, des armes personnelles des munitions, 24 avions de chasse F- 6 et F-7, 100 chars d'assaut, 144 missiles air-air, et quatre bateaux patrouilleurs. Ces développements indiqueraient la fin de la position officielle de neutralité de la Birmanie, et le début d'une politique prochinoise à Rangoon. Ce changement est préoccupant du point de vue de l'Inde, qui a une frontière commune avec la Birmanie. Les observateurs de la situation militaire en Birmanie indiquent que des armements chinois servent à renforcer la puissance de la junte militaire de Rangoon en augmentant la présence militaire de l'armée dans les pays, y compris le long de la frontière indienne ${ }^{21}$. Les transferts d'armements en Asie du Sud -Pakistan La plupart des armements chinois exportés vers l'Asie du Sud vont au Pakistan, principal allié de la Chine dans la région. Toutefois, d'autre pays de la région Afghanistan, Bangladesh Népal, Sri Lanka - ont acheté un nombre croissant d'armes à la Chine. Depuis les années soixante, la RPC qui a offert son aide militaire et économique dès la guerre indo-pakistanaise de septembre $1965^{22}$ a été un des principaux 
fournisseurs d'armement du Pakistan. Selon l'International Institute for Strategic Studies à Londres, les exportations d'armes chinoises vers le Pakistan ont commencé avec un accord de juillet 1966 portant sur une valeur de 120 millions de dollars : la Chine vendait des chars d'assaut T 59 des avions de chasse F-6, et des avions de bombardement II- $28^{23}$. Depuis, elle est devenue le plus sûr et le plus important fournisseur militaire du Pakistan où pendant un quart de siècle, elle a exporté tous les types de matériel : équipement et matériel individuel : munitions, armes personnelles, grenades à main, lance-fusées, transports blindés, chars, navires de guerre, avions militaires, missiles et technologie de missile, enfin la Chine a activement contribué au développement de l'arsenal nucléaire pakistanais. Après l'humiliante défaite que l'Inde a infligée au Pakistan en 1971, Pékin a décidé de moderniser complètement, quasiment gratuitement ou à l'aide d'offres extrêmement avantageuses, l'armée pakistanaise. Depuis cette date, la Chine qui est - rappelons-le - le fournisseur d'armements le plus fidèle du Pakistan, a exporté une grande quantité d'armements couvrant tous les secteurs : 1500 chars, 350 avions de chasse, 30 navires de guerre, et très récemment des missiles à moyenne portée, capables de porter une bombe atomique (voir le tableau 5). Il faut souligner tout particulièrement les projets de production commune d'armement, qui ont caractérisé les relations entre les deux pays ces dernières années. Par exemple, la RPC a aidé le Pakistan en construisant des usines pour entretenir et moderniser les avions de guerre F-6, F-7 et Q-5 Fantan; les deux pays coopèrent à la production d'un avion-école militaire, le Karakoram-8. En ce qui concerne les blindés, le Pakistan, grâce à l'aide chinoise, est en train de développer une capacité autonome de production d'une version moderne du char d'assaut chinois T-69. Cette version sera dotée d'un canon rayé de $105 \mathrm{~mm}$, d'un télémètre à laser, d'un système de direction de tir informatisé, d'un blindage renforcé, et d'un moteur plus puissant. Cette usine, capable de fabriquer 150 à 200 chars de ce type à partir de 1991, produira aussi des canons rayés de $105 \mathrm{~mm}$ qui doivent être montés sur les T 59 chinois de l'arsenal pakistanais. De plus toujours grâce à l'assistance chinoise, les militaires pakistanais sont en train de développer une version d'un missile portable sol-air conçu en Chine (copie du missile américain "Stinger"), et la missile chinois antichars ${ }^{24}$. "Hong Jian-8" (Flèche Rouge-8), missile chinois antichars(15). L'aspect le plus controversé des transferts militaires chinois au Pakistan demeure les armements non conventionnels et tout spécialement la coopération dans le domaine de la technologie nucléaire. Les dernières informations parvenues à l'Ouest montrent que la RPC fournit le Pakistan en uranium destiné aux processus d'enrichissement. La RPC appuie les plans d'armement nucléaire pakistanais et il est possible que Pékin ait permis aux Pakistanais de faire exploser une bombe atomique en Chine ${ }^{25}$ Grâce à cette assistance, le Pakistan aurait aujourd'hui la capacité de réaliser un certain nombre de bombes, et de plus, possèderait assez d'uranium "enrichi au niveau offensif" [weapons-grade] pour fabriquer dix bombes atomiques ${ }^{26}$. Lors de sa visite en novembre 1989 en Asie du Sud, le Premier ministre chinois Li Peng a offert de construire une centrale nucléaire de grandeur moyenne, et il a évoqué la possibilité de transférer au Pakistan un sous-marin d'attaque nucléaire ${ }^{27}$. Globalement, ces développements dans le domaine nucléaire liés aux progrès pakistanais dans le domaine de la technologie de missiles (c est le Pakistan qui assure la formation du personnel capable de mettre en oeuvre les missiles chinois CSS-2 vendus à l'Arabie Saoudite) montrent nettement la capacité potentielle de fabrication de missiles nucléaires au Pakistan. Bref, il ne faut pas sous-estimer l'ampleur de l'assistance militaire chinoise au Pakistan. Jusqu'au grand pacte d'assistance militaire de 1982 entre 
les Etats-Unis et le Pakistan, la Chine a été de loin le plus important pourvoyeur d'armements et de technologie militaire au Pakistan: elle a fourni un tiers de tous les armements exportés vers le Pakistan de 1966 à 1980, donc largement plus que les deux autres principaux fournisseurs, les Etats-Unis et la France. De nos jours, devant les progrès pakistanais dans le domaine nucléaire, Washington a supprimé son assistance militaire au Pakistan. Cette décision permet à la Chine de rester, à moyen terme, le fournisseur d'armements le plus important du Pakistan. - Afghanistan, Népal, Sri Lanka, Bangladesh La Chine entretient ausss des relations importantes en tant que fournisseur d'armements avec d'autres pays en Asie du Sud l'Afghanistan, le Népal, le Sri Lanka, le Bangladesh - circonstance aggravante du point de vue de l'Inde. Mais même si les niveaux quantitatifs et qualitatifs des armements exportés à ces clients restent encore relativement faible ces dernières années, il est net que la RPC essaye de renforcer ses positions d'exportatrice dans les pays de la région. En Afghanistan, pendant les années quatre-vingts, la Chine exportait des munitions, des projectiles, des lance-fusées, et des missiles portables antiaériens aux résistants antisoviétiques. Au Népal, les exportations militaires étaient relativement faibles : la Chine a fourni en 1988 dix batteries d'artillerie antiaérienne. Depuis 1989, les transferts d'armement chinois au Sri Lanka augmentent rapidement et s'ajoutent à d'autres armements livrés pendant les années soixante-dix/quatrevingts (pièces d'artillerie et canonnières). On dénombre cinq avions de transport $\mathrm{Y} 8$ et $\mathrm{Y} 12$, un certain nombre d'avions d'attaque à réaction $\mathrm{Q}-5$, un régiment de véhicules blindés d'infanterie motorisée $\mathrm{T}-85$, un régiment d'artillerie lourde à longue portée type 59/1, et deux canonnières modernisées de la classe "Shanghai". Cette croissance des exportations militaires chinoises, fait de Pékin en 1991 le premier fournisseur d'armements du Sri Lanka ${ }^{28}$. Au Bangladesh, les transferts chinois d'armements y ont été relativement importants ces dernières années. La RPC a fourni des armements dans les quatre secteurs principaux de la production militaire chinoise - avions, blindés/artillerie, missiles et vaisseaux de guerre. L'arsenal du Bangladesh comprend les armements chinois suivants : des avions de chasse F-7, des avions d'attaque Q-5, chars d'assaut T-59, des missiles HY2 (nommés "Silkworm" à l'Ouest), des frégates "Jianghu", et un sous-marin de la classe "Roméo".

Tableau 5

Les transferts d'armements traditionnels de la Chine vers le Pakistan (1965-1991) ${ }^{29}$

Les transferts d'armements en Asie du Sud-Ouest Pendant les années quatre-vingts, la quantité d'armements chinois exportée vers l'Asie du Sud-Ouest a augmenté rapidement. Cette région est devenue le premier débouché des livraisons militaires chinoises pendant cette période, puisque les exportations d'armes vers l'Irak, l'Iran et l'Arabie Saoudite y représentaient à elles seules les deux-tiers des armements chinois exportés de 1979 à $1988^{30}$. Mais, la Chine a envoyé des armements à d'autres pays de la région : Oman, Yémen et Syrie. -Iran, Irak Après la chute du Shah qui a entraîné l'arrêt brutal de l'aide militaire américaine à l'Iran, la RPC a commencé à vendre des armements à l'Iran - devenue paria aux yeux de l'Occident - et leurs relations sont devenues assez complexes: la Chine (avec la Corée du Nord) a fourni $70 \%$ des importations iraniennes d'armements pendant les années quatre-vingts ${ }^{31}$. Selon un accord passé entre les deux pays, la Chine a autorisé l'Iran à produire un missile chinois sol-sol (nommé "Oghab" chez les Iraniens). De tels accords de production sous licence sont pour les Chinois relativement rares, la RPC n'a passé de tels accords de production qu'avec ses clients les plus proches: la Corée du Nord, l'égypte, le Pakistan, la 
Thailande. Il est également possible que le Pakistan exporte prochainement des armements - conçus en Chine, mais fabriqués au Pakistan - vers l'Iran ce qui constituerait une "ligne de défense islamique" contre l'Inde en Asie du Sud ${ }^{32}$. En 1991, on trouvait dans la presse des commentaires accusant la Chine d'avoir aidé l'Iran à développer une capacité nucléaire. La Chine a nié ces allégations ${ }^{33}$, elle a seulement admis avoir fourni pendant les dix dernières années de la technologie nucléaire notamment un petit réacteur - à l'Iran dans un but pacifique de recherche médicale et scientifique. Les transferts d'armements traditionnels de la Chine vers le l'Iran ${ }^{34}$. Le commerce des armes entre la Chine et l'Irak s'est développé selon un schéma semblable. Vers la fin des années soixante-dix et le début des années quatre-vingts, au moment où l'URSS commençait à diminuer la quantité d'armes exportées vers l'Irak, la Chine commençait à livrer des armements et des pièces de rechanges compatibles avec les armes soviétiques (la Chine avait fait la même chose avec l'égypte pendant les années soixante-dix). L'exportation d'armements chinois vers l'Irak renforçait d'autre part les liens sino-arabes à travers un circuit d'échange à deux branches: la Chine vendait du matériel neuf à l'égypte; l'égypte revendait des armements chinois et soviétiques plus anciens à l'Irak. Avant l'invasion du Koweït, l'Irak a accumulé une vaste quantité de blindés et d'artillerie chinois : il est possible que l'Irak ait commandé au moins 2000 exemplaires du meilleur char chinois - le T-69 dont environ 600 auraient été livrés avant fin 1988; 700 chars d'assaut T 59, 650 transports blindés T 531, 720 pièces d'artillerie lourdes à longue portée $130 \mathrm{~mm}$ furent aussi livrées avant cette date. Fait remarquable, selon un rapport récent d'un groupe privé de recherche, le "Nuclear Control Group", de 1984 à 1986, la Chine et l'Irak, ont engagé une étude de faisabilité pour déterminer la possibilité de développer une centrale nucléaire en Irak. La RPC a nié le rapport avec véhémence ${ }^{35}$.

Pendant les années quatre-vingts, le commerce d'armements avec l'Irak et l'Iran a rapporté plus de 8 milliards de dollars à la Chine ce qui représente les trois-quarts des bénéfices tirés de ce secteur par la Chine ${ }^{36}$. La Chine envoyait à l'Iran et à l'Irak, une grande quantité d'armements de toute nature: des centaines d'avions, des milliers de chars, des transports blindés, des pièces d'artillerie, de nombreux missiles de plusieurs types (antiaérien, antichars, anti-navires), et fournissant une assistance militaire: entraînement, conseillers, munitions, et équipements personnels compris. En 1988, la RPC a même continué à fournir des armements aux deux pays après l'entrée en vigueur du cessez-le-feu entre l'Iran et l'Irak. Selon certains rapports, de nouveaux accords avaient été passés ; la RPC livrait encore quelques lanceurs et des missiles anti-navires (Silkworm), des lanceurs et des missiles antiaériens HQ-28. Un autre accord concernait l'usage "commun" d'un satellite de reconnaissance chinois" ${ }^{37}$. Par contre après l'invasion du Koweït en 1990, la Chine a officiellement suspendu ses exportations militaires à l'Irak. Néanmoins, des rapports laissent entendre que la coopération militaire continue entre la Chine et l'Irak. Selon l'un d'entre eux la Chine a continué à envoyer des munitions à l'Irak, et le grand conglomérat militaire chinois (China North Industries Company - NORINCO) a décidé de fournir à Bagdad des munitions et de l'hydrure lithinifère - un produit chimique utilisé en fabriquant des gaz innervant, du combustible de missile. Le ministre des Affaires étrangères chinois dément cette information ${ }^{38}$. Quoiqu'il en soit, après le cessez-le-feu les livraisons militaires chinoises vers l'Iran et l'Irak ont beaucoup diminué, et cela se traduit dans les statistiques où les exportations d'armements chinois passent des records de 1988 aux chiffres plus bas de 
1989/1990. Les tableaux 6 et 7 présentent avec plus de détails les transferts d'armements de la Chine à l'Iran et à l'Irak. Tableau 7

Les transferts d'armements traditionnels de la Chine vers le l'Irak ${ }^{39}$

Les transferts d'armements et la violence régionale Les exemples évoqués ci-dessus fournissent une première série d'analyses bilatérales des exportations d'armements chinois. Nous allons à partir de là essayer, dans les pages qui suivent, d'envisager d'une manière globale les relations entre les exportations d'armements chinois et la violence régionale, actuelle ou potentielle. Nous partirons d'une matrice construite à partir de quatre critères : armes, violence, expansion, diminution, et qu'on peut résumer à l'aide de quatre scénarios :

1 - les exportations d'armes contribuent à la violence régionale ;

2 - les exportations d'armes découragent la violence régionale ;

3 - la violence régionale contribue à l'exportation des armes ;

4 - la violence régionale décourage l'exportation d'armements.

Ces relations peuvent être formalisées dans le schéma ci-dessous. En répondant à cette question : Comment les cas étudiés dans la première partie de cet article s'intègrent-ils dans le schéma? nous comprendrons mieux, non seulement la politique chinoise d'exportations d'armes, mais encore le rôle des armes chinoises comme facteur développant ou freinant la violence régionale. Au départ, il faut admettre que le schéma proposé ne représente pas exactement les relations existant entre les armes exportées et la violence, la réalité n'est point aussi simple. Nous verrons notamment que dans le commerce international des armes, les situations sont mouvantes, qu'elles se chevauchent, qu'elles sont également obscures, ou difficiles à établir avec certitude. Pourtant, cette matrice est utile car elle offre une "charpente conceptuelle" à l'analyse qui aide à éclaircir et à délimiter les relations - souvent nébuleuses, toujours difficiles à saisir - entre les armes exportées et la violence régionale. Les exportations d'armements de la RPC vers la Thaillande et les Khmers rouges, vers le Pakistan, l'Iran et l'Irak que nous avons décrites dans la première partie - sont toutes suffisamment importantes et durables pour nous guider dans l'examen des quatre scénarios de notre matrice. Les armes exportées de la Chine contribuent à la violence régionale (scénario 3) Dans ce scénario, l'exportation d'armements chinois exacerbe les conflits. Cela signifie que les armements chinois engagés dans un conflit contribuent nettement et à long terme à sa prolongation et à son intensification et ce, à un niveau qui serait inenvisageable sans l'existence de ces armements. De tous les exemples que nous avons donné, les armements fournis aux Khmers rouges illustrent le mieux cette relation entre les armes et la violence régionale. Sans l'appui ferme de la chine, à partir du milieu des années soixante-dix, les Khmers rouges n'auraient jamais pu résister avec succès à l'armée vietnamienne et à celle de gouvernement cambodgien. Mais, ce cas est aussi un exemple de la nature inconstante de ces relations : la fourniture d'armes aux Khmers rouges aurait contribué à décourager la violence dans la mesure où elle a contribué à l'affaiblissement de la présence vietnamienne au Cambodge et dans la région. Il ne faut pas négliger le fait que les armes étaient fournies aux Khmers rouges gratuitement, et qu'elles faisaient partie d'une politique stratégique à long terme par laquelle Pékin cherchait surtout à protéger ses intérêts à la périphérie du sud de la Chine. Les armes exportées de la Chine découragent la violence régionale (scénario 2) Dans ce scénario, les armes exportées évitent l'épanouissement l'expansion ou 
l'intensification de la violence régionale. Dans le contexte des ventes chinoises, ce scénario se produit si la présence d'armements chinois contribue d'une manière significative à contenir ou prévenir la violence. Cela signifie que la violence éclaterait s'il n'y avait pas eu vente d'armements chinois. Parmi les cas étudiés, ce sont les transferts d'armements chinois au Pakistan qui illustrent le mieux ce scénario. Depuis l'année 1971, qui marque la défaite, le démembrement et l'humiliation du Pakistan par l'armée indienne, la Chine a pris sur elle de rebâtir la puissance militaire pakistanaise et d'annoncer nettement la résolution immuable de Pékin de protéger son allié de l'Asie du Sud. Si cette position n'avait pas existé, et en particulier s'il n'y avait pas eu une assistance fournie pour développer la capacité nucléaire pakistanaise, il est probable que le calme relatif existant dans la région n'aurait pas duré si longtemps. Si la situation stratégique de la région n'est pas entièrement stable, la présence des armements chinois contribue d'une manière significative au développement et au maintien de son équilibre politique. Cela s'est traduit pendant les vingt dernières années par une situation relativement calme, quand on la compare à la précédente décennie. Les exportations militaires chinoises vers la Thaïlande contribuent également à contenir l'expansion de violence, mais ce cas n'est pas aussi clair que celui du Pakistan. Le commerce d'armements sino-thaï remplit une double mission. Il servait à contenir l'expansion violente du Vietnam dans la région indochinoise, ce qui est l'objectif de la Chine, et, du point de vue thaïlandais, prévient un élargissement géographique de la guerre cambodgienne, c'est-à-dire les incursions sur le territoire thaïlandais des armées vietnamienne et cambodgienne. Les armements chinois exportés vers la Thaïlande ont donc bien atteint leur but. Mais l'armée thaïlandaise se fournissait à d'autres sources : les Etats-Unis, le Royaume-Uni l'Allemagne de l'Ouest, entre autres, lui ont vendu de vastes quantités d'armes. II est fort probable que ces exportations militaires à elles seules auraient contribué à empêcher l'implication de la Thaïlande dans la violence régionale. Dans une certaine mesure, la présence d'autres fournisseurs diminuait donc le rôle des armements chinois en décourageant la violence à l'égard de la Thaïlande. Néanmoins, il ne faut pas sous-estimer la présence hégémonique de la Chine dans la région. Dans les calculs faits par les expansionnistes de Hanoi, la prise en compte des liens solides existant entre la Chine et la Thaillande développés et fortifiés par le commerce d'armements - a contribué sans aucun doute à l'affaiblissement de la résolution des "stratégistes" vietnamiens, et finalement à la retraite de l'armée vietnamienne du Cambodge. Il est remarquable que dans les deux cas, Pakistan et Thaïlande, les armements chinois étaient fournis soit gratuitement, soit à "prix d'amis" (cela signifie avec un très fort rabais), ou à des conditions de remboursement favorables. Pour ces deux pays - ainsi que pour les Khmers rouges - les exportations d'armements chinois étaient un moyen au service d'une stratégie traditionnelle : la garantie des intérêts vitaux de la Chine à sa périphérie sud contre l'Inde et le Vietnam. La violence régionale contribue à l'exportation d'armements chinois (scénario 3) Dans ce scénario, le courant d'exportations des armes dépend de l'existence a priori d'un conflit. Ce scénario est réalisé quand les armements chinois sont attirés par un conflit qui existerait qu'il y ait des armes chinoises ou pas. Dans ce sens, les exportations d'armements chinois sont "réactives" ou "opportunistes" car les fournisseurs d'armements chinois profitent du déclenchement d'un conflit pour envoyer des armes. Les exportations d'armements chinois pendant la guerre Iran-Irak correspondent bien à ce scénario. Avant le commencement de ce conflit, la Chine n'avait jamais fourni d'armes à l'Iran et à l'Irak. De plus, la présence, ou l'absence 
d'armements chinois dans la région n'a eu - ou n'aurait eu - aucune incidence sur le déclenchement de la guerre. Fait encore plus important, la Chine étant devenu fournisseur des deux belligérants : la guerre Iran-Irak a correspondu à peu près au trois quarts des exportations d'armements de la Chine ${ }^{40}$ pendant les années quatre-vingts. L'exportation d'armements chinois dans le cadre de ce conflit nous montre clairement comment les scénarios peuvent évoluer. Pendant les premières années de la guerre, la violence attirait des armes chinoises. Mais quelques années plus tard, les armes de la RPC contribuaient à prolonger la violence entre l'Iran et l'Irak. Vers le milieu de la guerre, la Chine et la Corée du Nord ont soutenu l'Iran affaibli et ont fourni $70 \%$ des armements que les Iraniens importaient. A ce moment-là, la Chine donnait à l'Iran, soumise à l'embargo international sur les armements, des moyens militaires nécessaires pour continuer la lutte contre l'Irak.

La violence régionale décourage l'exportation d'armements chinois (scénario 4) Pour que ce scénario se produise, il faut qu'un fournisseur d'armes - pour certaines raisons craigne une condamnation internationale, une intensification de conflit, ou tout simplement son implication dans le conflit : il est alors dissuadé d'exporter ses armes vers une région où existent des rivalités ou des conflits ouverts. Tout en comprenant qu'il est virtuellement impossible de savoir de façon certaine si ces considérations ont effectivement limité les exportations militaires de la Chine, il nous semble que la réponse est non. Dans la plupart des cas que nous avons examinés, il semble que la Chine ne se soit préoccupée ni de la condamnation politique ni d'une expansion du conflit provoqué par ses exportations d'armes. Il existe des cas plus discutables : par exemple, pendant la guerre du Vietnam dans les années soixante/soixante-dix, Pékin est resté assez prudent dans ses livraisons d'armes à Hanoi car la Chine avait peur d'une confrontation directe avec l'Amérique. Cependant, la Chine fournissait d'importantes quantités d'armes au Nord-Vietnam. En analysant nos cas, il semble que la Chine ait rarement été découragée de vendre ses armes. Cette conclusion, et celles qui ont précédé, nous amènent à formuler des conclusions finales sur les rapports entre exportations d'armes chinoises et avenir de la violence régionale. Motifs et résultats Comme tous les facteurs internationaux, le commerce international n'est pas statique. Mais la prise en considération de cette mobilité du système international qui concerne bien sûr les exportations militaires chinoises, n'empêche pas de formuler quelques hypothèses situées dans un futur prévisible, sur les rapports entre transferts d'armements chinois et violence régionale. Si les déterminants nationaux et internationaux restent relativement stables, il semble que les résultats constatés dans le scénario 3, où les armes suivent la violence, définiront les relations entre les exportations militaires chinoises et la violence régionale. Bref, à cause de déterminants nationaux et internationaux de politique et de stratégie, il est probable que la Chine ne diminuera pas ses exportations d'armements dans un avenir proche. Déterminants nationaux Une étude récente portant sur la classe militaire chinoise ${ }^{41}$ montre que, pendant la dernière décennie, les exportations d'armements ont été de plus en plus décidées par des responsables d'entreprises d'armements semi-autonomes très influents, mais ne faisant pas officiellement partie du gouvernement national. De temps en temps, il semble que le pouvoir et les activités de ces entreprises soient indépendants du ministre des Affaires étrangères, du ministre de la Défense, et d'autres ministres et organisations de l'état et du Parti qui, cas typique, s'occupent d'exportations d'armements. Indépendantes, ne rendant des comptes qu'à elles-mêmes, ces entreprises se lancent dans le commerce des armes pour servir les intérêts 
personnels de dirigeants qui sont souvent des militaires très haut placés ou des proches du personnel politique. Ces intérêts ne correspondent pas nécessairement aux intérêts stratégiques ou politiques de l'état ou du Parti formulés par les ministres ou l'appareil du Parti. Il est possible que les ministres aujourd'hui ne contrôlent pas toujours la nature ou la direction de certaines transactions d'armes. Si cette analyse se révèle exacte, les exportations d'armements chinoises représenteront de plus en plus les intérêts des individus et des groupes de "bonne famille", et, de moins en moins les intérêts géopolitiques ou stratégiques de la RPC. Bref, les exportations "réactives", c'est-à-dire les exportations d'armements à but uniquement lucratif, où les armes suivent la violence, peuvent devenir la norme. Fournir des armes à une région où existe déjà un conflit l'exacerbera au lieu de le stabiliser. Ainsi, il est probable que les futures exportations d'armements de la RPC aboutiront à la situation prévue ci-dessus dans le scénario $3:$ une approche réactive et opportuniste où les fournisseurs chinois ne se préoccupent pas trop de considérations politiques et stratégiques et des implications à long terme de leurs décisions. Déterminants internationaux Quant aux déterminants internationaux, ils suggèrent aussi mais d'une manière différente - que la Chine continuera à vendre activement des armements comme prévu dans le scénario 3 . Il est remarquable qu'il y a quelques années la Chine, consciente de sa situation stratégique, était disposée à utiliser les ventes d'armes dans le but de protéger ses intérêts stratégiques et politiques. Les scénarios 1 et 2 sont ceux correspondant aux situations où la Chine s'occupait en priorité de ses intérêts stratégiques et politiques - on pense ici au Pakistan, à la Thaïlande, et aux Khmers rouges. Mais, dans la situation internationale actuelle, il faut nous demander si de graves menaces pèsent sur la Chine: l'URSS n'est plus une super puissance, le processus de paix est entamé au Cambodge, alors existe-t-il des motifs stratégiques pour pousser la Chine à fournir des armes dans le sens des scénarios 1 et 2 décrits ci-dessus ? On peut répondre : peu dans le futur prévisible, sauf peut-être la politique de l'Inde. De toute façon la Chine continuera à fournir des armes au Pakistan contre cette menace apparente. Il semble que les déterminants internationaux actuels rendent les situations prévues dans les scénarios 1 et 2 de plus en plus improbables. De toute façon, comme le montre son comportement passé, la Chine se montre très peu disposée à limiter volontairement ses exportations d'armements: sa réponse à de telles propositions est en général très défensive. Plus les autres grands fournisseurs diminuent la quantité d'armements exportés, plus la Chine augmente ses exportations d'armements. Tout récemment, la Chine semblait disposée à discuter avec les autres grandes puissances de la possibilité de limiter la prolifération des armements conventionnel et nucléaire ${ }^{42}$. Mais il n'est pas possible de connaître avec certitude les résultats tangibles de ces discussions, et même si elles ont eu des résultats I Comme le passé annonce toujours un peu le futur, nous savons que si des conflits régionaux éclatent, la tentation de fournir des armes aux combattants sera forte chez l'oligarchie chinoise c'est d'ailleurs la même chose chez les autres grandes puissances. En résumé, l'examen des déterminants, soit nationaux, soit internationaux, nous permet de conclure que la Chine continuera activement à vendre des armements dans le monde. Les décisions d'exportations d'armements chinois étant de plus en plus prises en fonction de motifs personnels et opportunistes, et de moins en moins le résultat d'intérêts et de considérations stratégiques et politiques, les armes chinoises ont de grandes chances de contribuer dans l'avenir au maintien et à l'expansion de la violence régionale. 


\section{NOTES}

1. Wu est cité dans "China defends Saudi missile sale", Financial Times, 7 avril 1988 (traduit par l'auteur); voir aussi "Shuo renjia, xian yong jingzi zhao zijia" (avant de critiquer quelqu'un, regardez-vous auparavant dans un mirroir), Renmin ribao, 12 juillet, 1991.

2. Nous employons indifféremment dans le texte, la RPC ou la Chine.

3. International Institute for strategic Studies, Strategic Survey, 1988-1989, Londres, IISS, 1989, p. 23.

4. Le tableau est tiré de statistiques de Michael Brozska et Thomas Ohlson, Arms Transfers to the Thirld World, 1971-1985, Oxford, Oxford University Press, 1987, appendices 4A et 4B ; Stockholm International Peace Research Institute, SIPRI YearBook 1991, Oxford University Press, 1991, tableau 7A2.

5. Michael Brozska et Thomas Ohlson, op. cit.

6. Dollars américains.

7. SIPRI YearBook 1991, tableau 7A2.

8. Modernisation d'un matériel par l'introduction de nouveaux systèmes d'armes sur une cellule ou changement des composants électroniques. La Chine peut ainsi introduire des composants fabriqués à l'Ouest dans ses productions.

9. Jane's Defence Weekly, 27 mai 1989, p. 1020 ; Aaron Karp, "Ballistic missile proliferation in the Third World, SIPRI YearBook 1989, pp. 303-304.

10. Début des années 1980, voir Anne Gilks et Gerald Segal, China and the Arms Trade, New York, St martin's Press, 1985 ; William Tow, "Arms Sales", in Gerald Segal et William Tow (eds), Chinese Defence Policy, Londres, MacMillan, 1984 ; Wei-chin Lee, The Birth of a Salesman : China as an Arms Supplier", Journal of Northeast Asian Studies, hiver 1987-88. Pour des recherches plus récentes, voir entre autres : John W. Lewis, Hua Di, Xue Litai, "Beijing's Defence establishment : Solving the Arms-export enigma", International Security, printemps 1991 ; R. Bates Gill, Fire of th eDragon : Arms, Influence, and Chinese Security Policy, Programme for Strategic and International Security Studies, Occasional Papers n 1/1991, genève, PSIS, 1991.

11. Voir International defense Review, mars 1989, p. 356 ; International Herald Tribune, 7 mai 1987

12. Defense \& Foreign Affairs Daily, 6 août 1987.

13. Jane's Defence Weekly, 21 juillet 1990, p. 81.

14. Jane's Defence Weekly, 7 janvier 1989, p. 13 ; Asian Defence Journal, mars 1989, p. 118. International Defense Intelligence, 12 juin 1989 ; "Political High-flier", Far Eastern Economic Review, 7 décembre 1989, p. 21.

15. Cf. Article plus détaillé sur le commerce des armes de la Chine vers la Thaïlande, $R$. Bates Gill, "China Looks to Thailand : exporting Arms, Exporting Influence", Asian Survey, juin 1991.

16. Tableau tiré du SIPRI Yearbook (plusieurs éditions) et Jane's Defence Weekly (plusieurs publications

17. voir Anne Gilks et Gerald Segal, China and, op. cit., tableau 10.

18. Jane's Defence Weekly, 13 octobre 1990, p. 681.

19. SIPRI Yearbook 1991, appendice 7B.

20. Jane's Defence Weekly, 15 septembre 1990, p. 459. 
21. "Lock and load : Chinese supply suggest SLORC digging, "Far Eastern Economic review, 13 septembre 1988, p. 28 ; "Allies In Isolation : Burmar and chCina move closer, Jane's Defence Weekly, 15 septembre, p. 475 SIPRI Yearbook 1990, appendice 7B 22. Citant les débats de l'Assemblée Nationale Pakistanaise après la guerre de septembre 1965, Gurnam Sigh, Sino-Pakistan Relations : The Ayub Era, Amritsar : Guru Nanak Dev University Press, 1987, pp. 213-214.

23. Citant les débats de l'Assemblée Nationale Pakistanaise après la guerre de septembre 1965, Gurnam Sigh, Sino-Pakistan Relations : The Ayub Era, Amritsar : Guru Nanak Dev University Press, 1987, pp. 213-214.

24. Ces développements concernant les relations militaires entre la RPC et le Pakistan sont détaillés dans International Defence Review, juin, 1989, pp. 764-767 ; Jane's Defence Weekly, 11 août 1990, p. 187 ; SIPRI Yearbook 1991, appendice 7B.

25. Yaacov Vertzberger, "The political Economy of Sino-Pakistani Relations", Asian Survey, mai 1983, p. 648.

26. Washington Post, 12 mai 1991.

27. "Friends old and new", far Eastern Economic Review, 22 février 1990, p. 13.

28. International Defence Review, février 1991, pp. 107-108.

29. Ce tableau est tiré de plusieurs sources : SIPRI, Arms Trade registers : The Arms Trade with the Third World, Stockholm, Almqvist \& Wiksell, 1975 ; Michael Brzoska et Thomas Ohlson, Arms Tranfers to the Third World, op. cit. appendice 1 ; SIPRI Yearbook 1991, appendice 7B.

30. Durant la période 1979-1983, les transferts vers l'Iran et l'Irak équivalaient à 50\% des exportations des armes chinoises (il n'y avait pas de transferts vers l'Arabie Saoudite à cette époque). De 1984 à 1988, les trasnfers vers l'Iran, l'Irak, et l'Arabie Saoudite représentaient quant à eux $79 \%$ de tous les transferts militaires chinois). U.S. Arms Control and Disarmament Agency, World Military Expenditures and Arms Tranfers, 1985, Washington, D. C., U.S. Government printing Office, 1990, tableau III. 31. Richard F. Grimmett, Trends in Coventional Arms Transfers to the Third World by Major Supplier, 1979-1986, Washington, D.C. : Congressional Research Service, 1988, p. 27.

32. International Defence Review, juin 1990, p. 622.

33. Washington Post, 26 juin 1991, Washington Post, 2 juillet 1991 ; Free Press Wire Services, 31 octobre 1991.

34. SIPRI Yearbook (plusieurs éditions) ; Michael Brzoska et Thomas Ohlson, Arms Tranfers to the Third World, 1971-1985.

35. Voir le rapport de l'Agence France Presse, "Iraq Nuclear Study Denied", Foreign Broadcast Information Service Daily Report, China, 3 juillet 1991, p. 7.

36. Eden Y. Woon, " Chinese Arms Sales and US-China Military relations", Asian Survey, juin 1989, p. 604.

37. SIPRI Yearbook 1990, appendice 7B. Concernant le satellite de reconnaissance, voir Defence \& Foreign Affairs, 19 juin 1989, p. 8.

38. "Who us ?" The Economist, 6 octobre 1990, p. 38. Voir aussi "China Tried to Sell Iraq Arms after embargo" Christian Science Monitor, 31 janvier 1991.

39. Le tableau est tiré de plusieurs sources : SIPRI Yearbook (plusieurs éditions); Michael Brzoska et Thomas Ohlson, Arms Tranfers to the Third World, 1971-1985. 40. Y. Woon, "Chinese Arms Sales and US-China Military relations", op. cit. D'autres observateurs du commerce international d'armements affirment que l'exportation d'armes chinoises vers l'Iran et l'Irak de 19791988 représentait 55\% de la totalité des 
armements en provenance de la Chine pendant cette période. U.S. Arms Control and Disarmament Agency, World Military Expenditures and Arms Tranfers 1985, Washington, D. C. : U.S. Government Printing Office, 1985, tableau III ; . U.S. Arms Control and Disarmament Agency, World Military Expenditures and Arms Tranfers 1989, Washington, D. C. : U.S. Government Printing Office, 1989, tableau III, . U.S. Arms Control and Disarmament Agency, World Military Expenditures and Arms Tranfers 1990, Washington, D. C. : U.S. Government Printing Office, 1990, tableau III.

41. John W. Lewis, Hua Di, Xue Litai, "Beijing's Defence establishment : Solving the Arms-export enigma", International Security, printemps 1991.

42. The Wasington Post, 8 juin 1991 ; The New York Times, 20 octobre 1991.Webmaster

\section{RÉSUMÉS}

La Chine est un fournisseur de technologie et de matériel militaires, conventionnels et nucléaires, de plus en plus important. La première partie de l'article examine à travers les exemples de la Thaïlande, du Pakistan, de l'Iran, de l'lrak... les caractéristiques de ce commerce depuis la fondation de la RPC. A partir de trois sites géográphiques - Asie du Sud-Ouest, Asie du Sud et Asie du Sud-Est - où les conflits sont nombreux. La deuxième partie de l'article tente de formaliser les liens entré l'exportation d'armes ét lá viólénce régionale. L'auteur conclut en suggérant que les déterminants internes et internationaux provoqueront dans un avenir prévisible une hausse des exportations d'armes : d'une part les armes de la RPC sont attirées vers des régions de tensions et de conflits (en Asie du Sud-Ouest par exemple) et d'autre part la RPC continue d'utiliser les exportations d'armes dans un but stratégique (Asie du Sud et du Sud-Est).

Facing the growth of the PRC as a provider of military-related technology and hardware - both conventional and nuclear -, we are compelled to consider the relationship between Chinese arms exports and regional violence. By considering the PRC's arms exports to three regions of tension and conflict - Southwest Asia, South Asia, and Southeast Asia - we may then gain insight into the relationship between Chinese arms and the prospects for regional violence. The article concludes by suggesting that due to international and domestic determinants, the PRC's arms exports in the foreseeable future are likely to expand as [1] the PRC's arms are drawn into regions of tension and conflict (as in Southwest Asia), and [2] as the PRC continues to use weapon exports to enhance its strategic interests (as in South and Southeast Asia).

\section{INDEX}

Index géographique : Asie du Sud-Est, Chine, Iran

Mots-clés : armes et armement, violence 
AUTEUR

R. BATES GILL

Professeur de science politique, spécialiste de l'Asie, à Lynchburg College, Virginia. 